
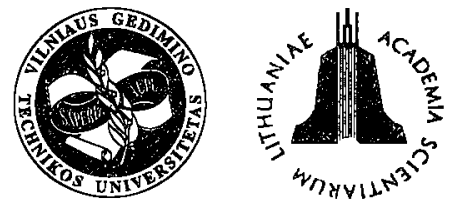

ISSN 1648-4142 TRANSPORT

http:/www.vtu.lt/english/editions

\title{
THE STUDY OF THE STABILITY OF ASPHALT CONCRETE MIXTURE COMPOSITION OPTIMIZATION MATHEMATICAL MODELS USING IMITATIVE SIMULATION
}

\author{
Valentinas Podviezko ${ }^{1}$, Henrikas Sivilevičius ${ }^{2}$ \\ ${ }^{1}$ Dept of Mathematical Statistics, Vilnius GediminasTechnical University, Lithuania, E-mail: stm@,st.vtu.lt \\ ${ }^{2}$ Dept of Transport Technologies, Vilnius Gediminas Technical University, \\ Lithuania,E-mail: henrikas@ti.vtu.lt,fax: (370-2)69-97-05
}

Received: 200306 03; accepted: 20031114

\begin{abstract}
The method of statistical imitative simulation (Monte-Carlo) was used to simulate the heterogeneity of the produced asphalt concrete mixture (ACM) mineral part grading. The stability of optimisation of mathematical models of ACM composition developed by us was tested by a computer using the theory of this method application. Average values $a_{i j}$ and their average standard deviations $\sigma_{i j}$ of seven $\left(A_{j}=7\right)$ finally batched aggregate partial residues on control sieves $i(i=9)$ were set for research. Imported filler $A_{1}$ and reclaimed dust $A_{2}$ were replaced by their mixture $\bar{A}_{1}$ when the accepted ratio of these materials masses $\lambda$ is 1,2 and 3 . The maximum $\left(A_{\max }\right)$ and minimum

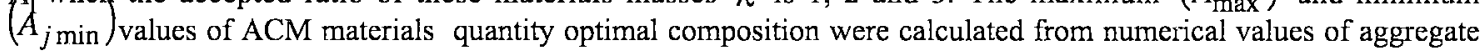
with different heterogeneity ((minimum $-\sigma_{i j \min }$, medium $-\sigma_{i j v i d}$, maximum $-\sigma_{i j \max }$ ) increasing the number of computer imitations $\mathrm{N}(100,300,500,1000,5000,10000)$. The graphs of the difference between the calculated maximum and minimum values of optimal quantity of aggregate dependence $\Delta A_{j}$ on the imitation number $N$ are presented. Calculation results proved the sufficient stability and practical application of the used optimization mathematical model to forecast ACM heterogeneity, when the heterogeneity and optimal quantity of the aggregate used in the mixture are known.
\end{abstract}

Keywords: asphalt concrete mixture, production technology, imitative simulation, model stability, Monte-Carlo method.

\section{Introduction}

Any mathematical model can be applied in practice as long as all other main parameters of the model are stable, i.e. when minor fluctuation of each argument in the model corresponds to the minor fluctuation of the characteristics (result) under investigation. The model stability may be tested by repeating the same experiment a lot of times, by slightly changing the values of model parameters and analyzing the variation of the obtained result after each change. In practice it is difficult to repeat the same experiment a lot of times since it takes a lot of time and costs a lot. To increase the data dependability the number of experiments shall be increased considerably.

To test the stability a well-known imitative simulation model (Monte-Carlo) may be applied [1-4].

The advantage of the stochastic imitative simulation is that not the experiment itself but the mathematical model of the experiment description is carried out (repeated). Thus, we obtain random, but possible (probable) values of each constituent, which depend on the parameter probable distribution and the main value of the calculated corresponding characteristics, i.e. mathematical model solution. Having a set of solutions the possible result fluctuation limits and empirical distribution are identified.

To design the asphalt concrete mixture (ACM) composition, methods [5-8] based on various principles, enabling to obtain different results, are used.

To produce ACM of the highest quality, conforming to the requirements of Construction Recommendations R 35-01, the mixture composition optimization mathematical model was developed and applied in practice $[9,10]$. The original calculation showed [9] that the calculated mass quantity of one of the finest aggregates (reclaimed dust $-A_{2}$ ) is negative: $A_{2}=-0,0552$. The result showed that in some cases the calculation model may be unstable. When producing ACM the aggregates (imported filler $\mathrm{Mm}$ and reclaimed dust $\mathrm{Md}$ ) may be interchanged decreasing the number of one material and increasing the quantity of another material the same amount. To identify the practical required quantity of the finest aggregates and to avoid a negative result the optimization model [10] with a different set ratio coefficient 2 of these aggregates was developed. 
When mass ratio $\lambda=A_{1} / A_{2} f$ multi-dispersive aggregates is preset by an asphalt concrete mixing plant operator in the batcher handling computer program, the technological process of the mixture production corresponds to the real scenario of necessary actions. The reclaimed dust which is constantly accumulated in a container is frequently used producing ACM. Therefore, the mass of its batches shall be sufficient to avoid its shortage or excess in the course of time.

The materials used producing ACM are heterogeneous [11]. During a working shift their grading fluctuates within wide limits which are shown by the factual values of average quadratic deviations of full passing through control sieves. Due to a segregation processes in ACMP batcher sections hot sieved fractions are not only more contaminated by by-grains, but also less homogeneous than an original imported filler. When heterogeneous finally batched aggregate is used, heterogeneous ACM is produced. Greater fluctuations of the produced ACM components quantity are influenced by greater fluctuations of the used aggregate grading. The curve of the designed optimal grading of ACM aggregate part fluctuates (slides) within a certain interval due to the heterogeneity of the used aggregate. When extremely heterogeneous aggregate is used, a case when the optimal average curve of the designed ACM, obtained from their grading means, conforms to the standard requirements (is within standard norms) may occur. However, in a separate case, due to the heterogeneity of materials, the maximum and minimum values of the mixture curve exceed the standard limits of curves. The methods of identifying the interval width of curves distribution have not been studied a lot.

The aim of this research is to test the stability of the mathematical optimization model of asphalt concrete mixture composition selection developed by us $[9,10]$ using the statistical imitative simulation.

\section{The Application Principles of Imitative Simulation}

When simulating the impact of $\mathrm{ACM}$ production technological process (operations) parameters variation on the homogeneity of its characteristics, we apply the optimization mathematical model $[9,10]$ ACM contains separate aggregates $A_{j}(j=1, \ldots, m)$, and each material contains three mineral components (crushed stone, sand and mineral powder) or more than three separate narrow (or wide) fractions. The grading of all mineral materials $j$ is identified at the beginning of ACM production technological process, i.e. before starting to produce it in ACMP. It is tested when characteristics of other original aggregate and (or) parameters of ACM production technological process change.

Most frequently partial residues $a_{i j}$ on each $i$ con- trol sieve $(i=1, \ldots, k)$ or and full passing through these sieves $p_{i j}$ are known (Table 1).

Aggregate heterogeneity is shown by average s quadratic deviations o-, of partial residues on control sieves (Table 2).

Optimal composition of ACM of the preset mark, which conforms to the composition specified in standards $[11,12]$ best, shall be produced from and more) original $r$ finally batched aggregate $A_{j}$ taking their grading into account ( $k$ partial residues on control sieves, $k=9$ ). Out of all possible ACM composition variants to be produced, the only best material mass ratio percentage, which is calculated using the least square method, is recommended. Here the condition that the summative difference square of calculated (project) $Z_{i}$, and set (standard) $Z_{o i}$ partial residues on control sieves $a_{i j}$ or full passing $p_{i j}$ through respective control sieves for all control sieves $k(i=1, \ldots, k)$ shall be the least.

Till now the determined mathematical model was applied to calculate the mass ratio percentage of materials used for asphalt concrete o any other mixture production when the values of all materials project quantity $A_{j}$ used for ACM production acquired a separate concrete value. Therefore, the solution of the optimization model showing the best selected quantity of materials is also concrete and unambiguous. The imitation of $\mathrm{ACM}$ production stochastic technological process provides a possibility to repeat the process under investigation a number of times, by setting not only the maximum, medium, and minimum values of aggregate grading but also separate random values, complying with the reality, changing within these limits due to the heterogeneity of materials; i.e, to obtain various their combinations. It also provides a possibility to replace a concrete single case by a real case repeated a number of times, by revealing the worst, average and the best quality of the mixture and the probability of their existance. Therefore, the optimization methodology presented in the research enables to apply the stochastic model instead of the determined one. Therefore, the data from Table 2 are used.

The main causes which impact on the instability of the results calculation are as follows:

1. Aggregates used for ACM production are heterogeneous due to the segregation in ACMP batcher sections [13]. The grading of hot fractions screened through ACMP technological sieves depends not only on the original cold aggregate and the grading of its mixture, but also on the changing technological parameters of the sieving process. Hot fractions still segregate in batcher sections. When taking samples to identify the factual grading, but selecting the required number and mass of samples inappropriately as well as applying improper sample taking methodology, additional errors are made. The impact of these factors on the heterogeneity of a finally batched aggregate is shown by values of average quadratic deviations (Table 2). 
2. When identifying the grading of all aggregate $j$ in a laboratory, errors of separate sample mixing, average sample reduction to testing sample and sieved residues on control sieves weighing are made, which depend on the accuracy of scales and proper actions of the laboratory specialist. Most frequently, the materials heterogeneity, sample taking and testing errors have normal distribution.

3. When calculating the composition of the selected mark ACM from its permitted lower $T_{A}=a_{i 0 \mathrm{~min}}$, and upper $T_{V}=a_{i 0 \max }$ values, interval mean $a_{i 0}$, is taken; although the composition of the mixture may vary within the interval $\left(T_{A}, T_{V}\right)$ and it is distributed according to normal distribution.

\section{The Sequence of Imitative Simulation}

The following sequence of statistical imitative simulation may be applied in research:
1. The mathematical model under investigation $U=F(X, Y, \ldots, Z)$ ( $U$ may be a vector as well) depends on random sizes with relevant distribution of parameters $X, Y, \ldots, Z$.

2. When a statistical sample $x_{1}, x_{2}, \ldots x_{n}$, of each parameter (e.g. $X$ ) is used before the application of the imitative simulation, we test the statistical non-parametral hypothesis on the theoretical distribution of parameter $X$, having applied one of compatibility criteria (Pirson, Kolmogorov, Smirnov or others).

3. We generate random values $\xi_{1}, \xi_{2}, \ldots, \xi_{n}$ comparatively high) of uniform distribution $N$ within interval $[0,1]$ with a computer generator and a relevant standard program or a table of random sizes.

4. When theoretical distribution function $F(x)=P(X<x) \quad(0 \leq F(x) \leq 1)$ of random size $X$ is used, from equation $\xi=F(x)$, having inserted generated numbers $\xi_{j}(j=\overline{1, N})$, relevant random values $N$ of size $X \quad \tilde{x}_{1}, \tilde{x}_{2}, \ldots, \tilde{x}_{N}$ are obtained applying inverse

Table 1. Matrix of mass percentage average means of aggregate partial residues on control sieves

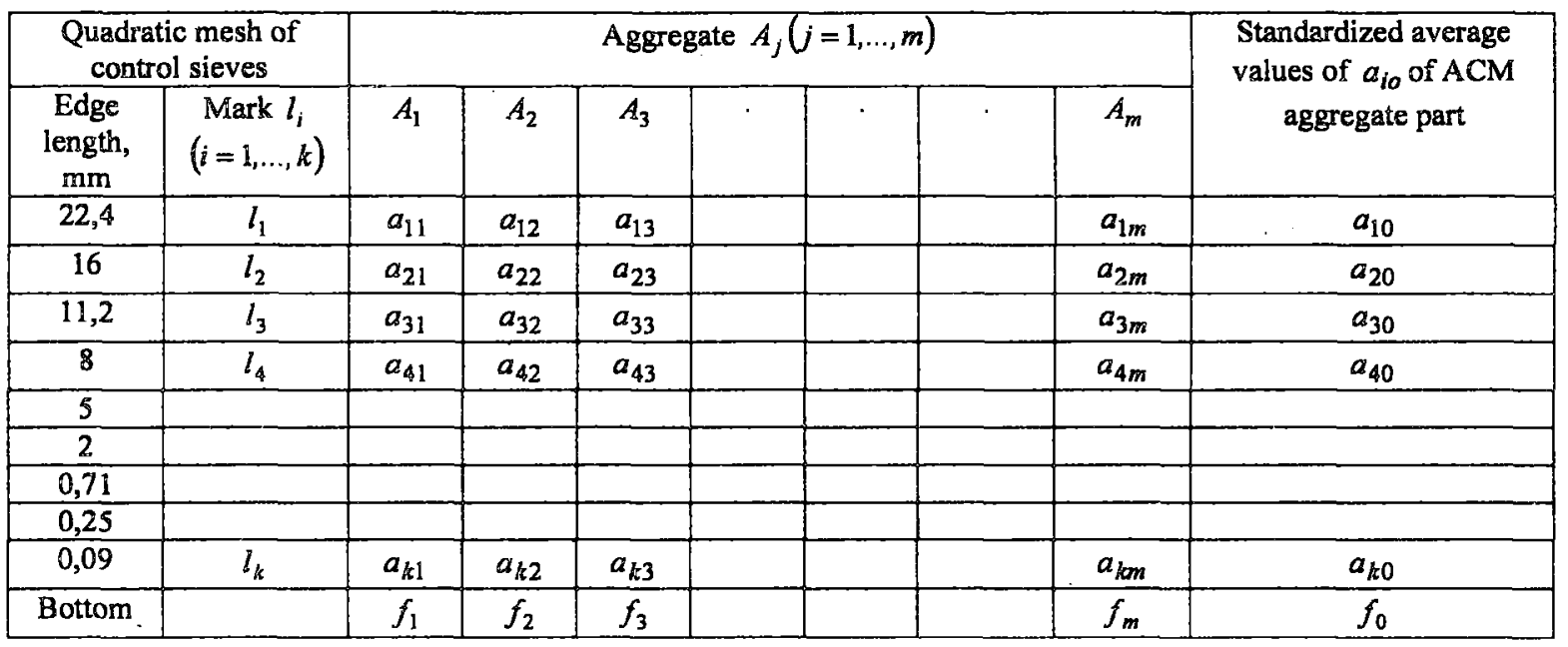

Table 2. Matrix of mass percentage average means of aggregate partial residues on control sieves

\begin{tabular}{|c|c|c|c|c|c|c|c|c|c|}
\hline \multicolumn{2}{|c|}{$\begin{array}{c}\text { Quadratic mesh of } \\
\text { control sieves }\end{array}$} & \multicolumn{7}{|c|}{ Aggregate $A_{j}(j=1, \ldots, m)$} & $\begin{array}{c}\text { Permitted values } \sigma_{i o} \\
\text { of ACM aggregate part }\end{array}$ \\
\hline $\begin{array}{c}\text { Edge } \\
\text { length, } \\
\text { mm }\end{array}$ & $\begin{array}{c}\text { Mark } l_{i} \\
(i=1, \ldots, k)\end{array}$ & $A_{1}$ & $A_{2}$ & $A_{3}$ & $\cdot$ & $\cdot$ & & $A_{m}$ & \\
\hline 22,4 & $l_{1}$ & $\sigma_{11}$ & $\sigma_{12}$ & $\sigma_{13}$ & & & & $\sigma_{1 m}$ & \multirow{2}{*}{ Not standardized } \\
\hline 16 & $l_{2}$ & $\sigma_{21}$ & $\sigma_{22}$ & $\sigma_{23}$ & & & & $\sigma_{2 m}$ \\
\hline 11,2 & $l_{3}$ & $\sigma_{31}$ & $\sigma_{32}$ & $\sigma_{33}$ & & & & $\sigma_{3 m}$ \\
\hline 8 & $l_{4}$ & $\sigma_{41}$ & $\sigma_{42}$ & $\sigma_{43}$ & & & & $\sigma_{4 m}$ \\
\hline 5 & & & & & & & & \\
\hline 2 & & & & & & & & \\
\hline 0,71 & & & & & & & & & \\
\hline 0,25 & & & $\sigma_{k 1}$ & $\sigma_{k 2}$ & $\sigma_{k 3}$ & & & & $\sigma_{l m}$ \\
\hline 0,09 & $l_{k}$ & $\sigma_{f 1}$ & $\sigma_{f 2}$ & $\sigma_{f 3}$ & & & & $\sigma_{f m}$ \\
\hline Bottom & & & & & & & & \\
\hline
\end{tabular}


$F(x)$ function $x=F^{-1}(\xi)$.

5. In practice normal $X$ distribution $N(a ; \sigma)$ is used most frequently; here $a$-mean, $\sigma$-average standard deviation. As it is known in this case it is impossible to calculate the distribution function $F(x)$ (integral) as well as to solve equation $x=F^{-1}(\xi)$. In this case standard computer procedure may be applied and if this procedure is missing, one of the transformations uniform distribution $\xi$ to normal $X$ applying, for example, one of algorithms $[2,3]$ is:

$$
x=x+\sigma_{x} \cdot\left(\frac{\sum_{j=1}^{m} \xi_{j}-\frac{m}{2}}{\sqrt{\frac{m}{12}}}\right),
$$

when $x$ and $\sigma_{x}$ are means of normal random size $X$ mean and a standard deviation, $m$ is the number of generated uniform distribution random sizes $\xi_{1}, \xi_{2}, \ldots, \xi_{m}$. When $m=12$, we obtain a separate case

$$
x=x+\sigma_{x}\left(\sum_{j=1}^{12} \xi_{j}-6\right) .
$$

6. Values of other parameter $Y, \ldots, Z$ random sizes $s$ be generated analogously, and values of each set $\tilde{x}_{j}, \tilde{y}_{j}, \ldots, \tilde{z}_{j}(j=1, N)$ shall be inserted into the model under investigation $U=F(x, y, \ldots, z)$. In this case we obtain $U$ values $\tilde{u}_{1}, \tilde{u}_{2}, \ldots, \tilde{u}_{N}$ of result $N$.

7. If the theoretical distribution of random size $X$ is not known or cannot be identified, but $X$ possible fluctuation interval $[a, b]=\left[X_{\min }, X_{\max }\right]$ is known, uniform distribution values $\xi_{j}(j=1, N)$ in interval $[0,1]$ may be rearranged into corresponding uniform distribution values $x_{j}=a+(b-a) \cdot \xi_{j}$ in interval $[a, b]$.

8 . When generating values $x, y, \ldots, z$ of different parameters or solving the task again, a new non-recurring sequence $\xi_{1}, \xi_{2}, \ldots, \xi_{N}$ as in a real experiment may be always obtained.

\section{Imitative Simulation of ACM Production Techno- logical Process}

When imitating the grading of the aggregate obtained in a laboratory is used (Table 3 ). We present imitative simulation as an example, where we took the means of finally batched aggregate partial residues on control sieves and their set theoretical a standard deviations showing minimum $\left(\sigma_{i j \text { min }} \int_{\text {medium }}\left(\sigma_{i j \text { men }}\right)\right.$ and maximum $\left(\sigma_{i j \max }\right)$ heterogeneity obtained from [10] (Table 4). The empiric distribution $A_{j}$ of all components $a_{i j}$ quantity in the aggregate was identified by experiments and it was found out that the quanity of a component on each sieve $i$ has a normal distribution with corresponding mean $a_{i j}$ of parameters and average standard deviation $\sigma_{i j}$. When imitating the process for each sieve $(i=1, \ldots, 9)$, we obtain random partial residues $a_{i j}$ of all aggregate $A_{j}(j=3, \ldots, 7)$ using standard programs of generating random numbers of the normal distribution with parameters $a_{i j}$ and $\sigma_{i j}$.

Corresponding values $f_{j}$ of each material $A_{j}$, i.e. partial residue with grains less than $0,09 \mathrm{~mm}$ of aggregate $j$ screened to the bottom of the sieve are calculated. Optimization task [14] is solved and optimal quantities of aggregates $A_{j}$ are obtained from the original data table (Table 3 ).

The process of calculating the optimal quantity in per cent of aggregate mass is imitated $N$ times using formulae (1) and (2). When calculating, maximum $\left(A_{j \text { max }}\right)$ and minimum $\left(A_{j_{\min }}\right)$ values of all materials $A_{j}$ quantity in unit parts are identified (Table 5). The (2) table contains only the data obtained from medium $\sigma_{i j}$ vid values when $\lambda=1,2,3$.

The sum of aggregate optimal quantity values $A_{j \min }$ for any number $N$ of imitations is less than 100 000 , and maximum values $A_{j \max }$ - more than 100000 . The modulus of optimal minimum quantity deviation $A_{j \text { min }}$ from mean $A_{j}$ is not the same as with maximum deviation $A_{j \max }$ from modulus $A_{j}$.

Calculation data of the difference of dependences $\Delta A_{j}=A_{j \max }-A_{j \min }$ from imitation number $N$ and graphs drawn according to them (Fig) show that when $N$ is increased (from 100 to 10000 ), values $\Delta A_{j}$ increase considerably at the beginning, and after a certain number of $N$, they almost do not change. Such number $N$ when ordinates of function $\Delta A_{j}=f(N)$ reach maximum stable values may be considered sufficient when simulating $\mathrm{ACM}$ heterogeneity.

The maximum $\left(A_{j \max }\right)$ and minimum $\left(A_{j \min }\right)$ values for set everage standard deviations $\sigma_{i j}$ vid of materials partial residues of each aggregate optimal quanitity ACM calculated by imitation vary inconsiderably (absolutely less than 2 mass in \%). When the aggregate heterogeneity ( $\sigma_{i j}$ values) increases, difference $\Delta A_{j}$ of optimal maximum $A_{j \max }$ and minimum $A_{j \text { min }}$ quantity in $\mathrm{AC}$ mixture increases. When extremely heterogeneous materials are used (most frequently due to segregation in ACMP hot batcher sections), difference $\Delta A_{j}$ of their optimal quantity increases. It can be so big that separate values of the designed $\mathrm{ACM}$ grading full passing through control sieves exceed tolerance limits of R 35-0 1 .

\section{Conclusions}

1. A new asphalt concrete mixture (ACM) grading designing method which is based not on the determined calculation of optimal quantity of an aggregate according to the arithmetic means of their partial residues on 


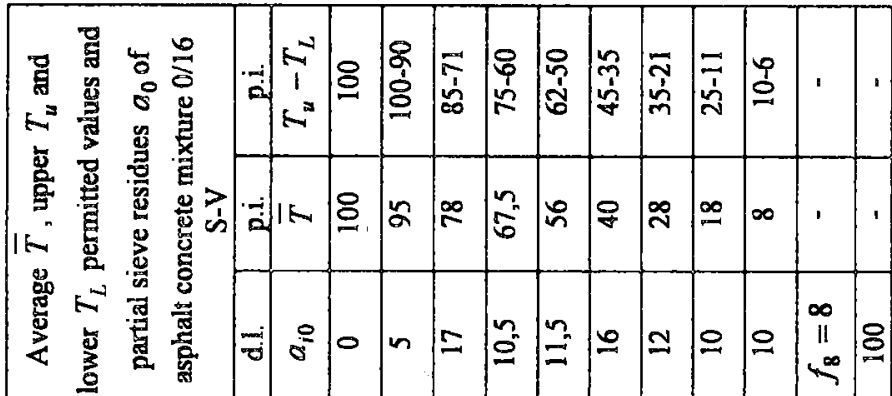

\begin{tabular}{|c|c|c|c|c|c|c|c|c|c|c|c|}
\hline \multirow{2}{*}{ 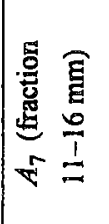 } & a & - & $\approx \Sigma$ & $m$ & $\tilde{m}$ & $\approx$ & $\overbrace{\delta}$ & 0 & 0 & 0 & \\
\hline & $-\overrightarrow{0}$ & $\frac{5}{5}$ & 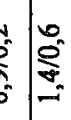 & 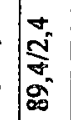 & $\mid \begin{array}{l}\infty \\
0 \\
0 \\
0 \\
0 \\
0\end{array}$ & 离 & 告 & 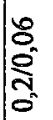 & & 잉 & $\begin{array}{l}0 \\
\text { II } \\
5\end{array}$ \\
\hline
\end{tabular}

\begin{tabular}{|c|c|c|c|c|c|c|c|c|c|c|c|c|}
\hline \multirow{2}{*}{ 愙 } & 8 & & & बे & $\ddot{\alpha}$ & $\stackrel{m}{=}$ & लू & $10^{\circ}$ & $\overline{0}$ & 0 & 10 & \\
\hline & ते & $\frac{0^{\circ}}{0^{\circ}}$ & 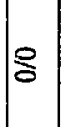 & $\begin{array}{l}\tilde{\delta} \\
\delta \\
0 \\
0 \\
0 \\
0 \\
0\end{array}$ & $\begin{array}{l}\infty \\
\delta \\
0 \\
0 \\
0\end{array}$ & $\mid \begin{array}{l}\infty \\
\infty \\
\infty \\
\infty \\
\infty\end{array}$ & $\mid \begin{array}{l}\infty \\
\sum^{0} \\
\infty\end{array}$ & & $\mid \begin{array}{l}0 \\
0 \\
0 \\
0 \\
0 \\
0\end{array}$ & $\mid \begin{array}{c}\delta \\
\delta \\
0 \\
0 \\
0\end{array}$ & 응 & \\
\hline
\end{tabular}

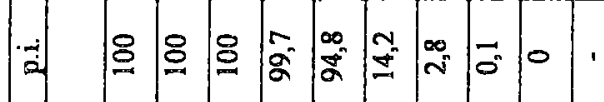

\begin{tabular}{|c|c|c|c|c|c|c|c|c|c|c|c|c|}
\hline \multirow{2}{*}{ 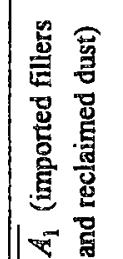 } & 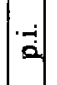 & & 8 & $\stackrel{8}{ }$ & 음 & 8 & 8 & 8 & $\begin{array}{l}\tilde{\alpha} \\
\alpha \\
\alpha\end{array}$ & $\frac{0}{2}$ & 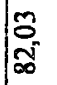 & \\
\hline & & $\frac{\sigma^{-}}{0}$ & 8 & 8 & 8 & 8 & 8 & 8 & 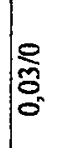 & 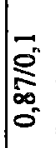 & 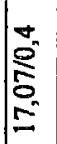 & 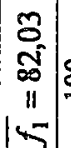 \\
\hline
\end{tabular}

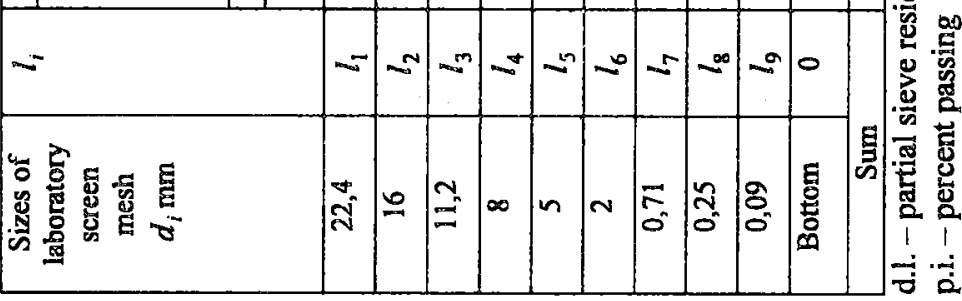


Table 4. Average standard deviations

\begin{tabular}{|c|c|c|c|c|c|c|c|}
\hline \multirow{2}{*}{$\sigma_{i j}$} & \multirow{2}{*}{$\begin{array}{c}\text { Sieve } \\
\text { mesh } \\
\text { size } \\
\text { mm }\end{array}$} & \multicolumn{6}{|c|}{ Mark of aggregate } \\
\hline & & $\bar{A}_{1}$ & $A_{3}$ & $A_{4}$ & $A_{5}$ & $A_{6}$ & $A_{7}$ \\
\hline \multirow[t]{9}{*}{ Min } & 22,4 & 0 & 0 & 0 & 0 & 0 & 0,1 \\
\hline & 16 & 0 & 0 & 0 & 0 & 0,01 & 0,3 \\
\hline & 11,2 & 0 & 0 & 0 & 0 & 0,2 & 1,2 \\
\hline & 8 & 0 & 0 & 0,01 & 0,03 & 0,9 & 0,4 \\
\hline & 5 & 0 & 0,025 & 0,04 & 0,7 & 0,4 & 0,2 \\
\hline & 2 & 0 & 0,05 & 0,5 & 0,15 & 0,1 & 0,1 \\
\hline & 0,71 & 0 & 0,125 & 0,12 & 0,08 & 0,02 & 0,03 \\
\hline & 0,25 & 0,05 & 0,15 & 0,02 & 0,01 & 0,01 & 0 \\
\hline & 0.09 & 0,2 & 0,1 & 0,01 & 0 & 0 & 0 \\
\hline \multirow[t]{9}{*}{ Med } & 22,4 & 0 & 0 & 0 & 0 & 0 & 0,2 \\
\hline & 16 & 0 & 0 & 0 & 0 & 0,02 & 0,6 \\
\hline & 11,2 & 0 & 0 & 0 & 0 & 0,4 & 2,4 \\
\hline & 8 & 0 & 0 & 0,02 & 0,06 & 1,8 & $\overline{0,8}$ \\
\hline & 5 & 0 & 0,05 & 0,08 & 1,4 & 0,8 & 0,4 \\
\hline & 2 & 0 & 0,1 & 1,0 & 0,3 & 0,2 & 0,2 \\
\hline & 0,71 & 0 & 0,25 & 0,24 & 0,16 & 0,04 & 0,06 \\
\hline & 0,25 & 0,1 & 0,3 & 0,04 & 0,02 & 0,02 & 0 \\
\hline & 0,09 & 0,4 & 0,2 & 0,02 & 0 & 0 & 0 \\
\hline \multirow[t]{9}{*}{$\operatorname{Max}$} & 22,4 & 0 & 0 & 0 & 0 & 0 & 0,4 \\
\hline & 16 & 0 & 0 & 0 & 0 & 0,04 & 1,2 \\
\hline & 11,2 & 0 & 0 & 0 & 0 & 0,8 & 4,8 \\
\hline & 8 & 0 & 0 & 0,04 & 0,12 & 3,6 & 1,6 \\
\hline & 5 & 0 & 0,1 & 0,16 & 2,8 & 1,6 & 0,8 \\
\hline & 2 & 0 & 0,2 & 2,0 & 0,6 & 0,4 & 0,4 \\
\hline & 0,71 & 0 & 0,5 & 0,48 & 0,32 & 0,08 & 0,12 \\
\hline & 0,25 & 0,2 & 0,7 & 0,08 & 0,04 & 0,04 & 0 \\
\hline & 0,09 & 0,8 & 0,4 & 0,04 & 0 & 0 & 0 \\
\hline
\end{tabular}

a)

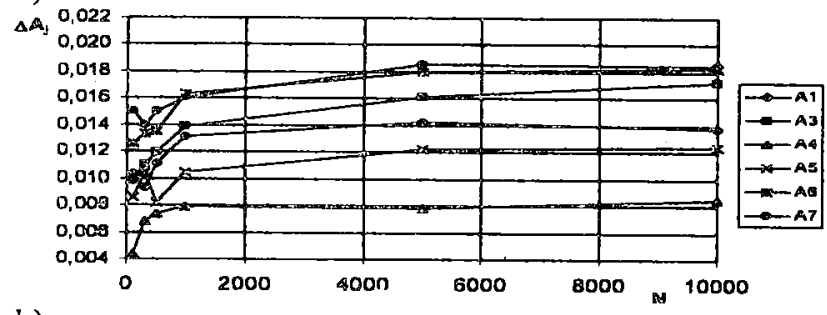

b)

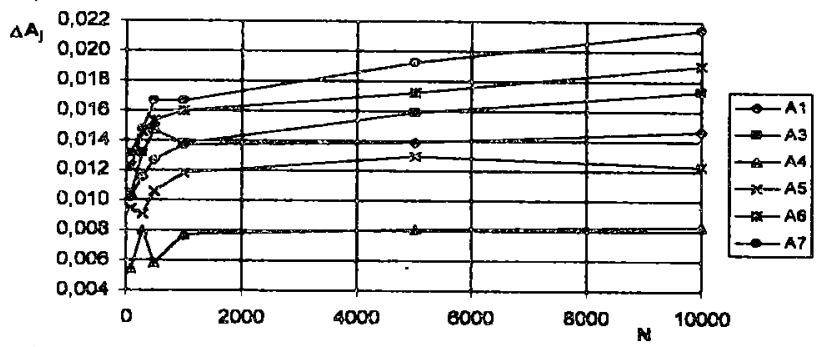

c)

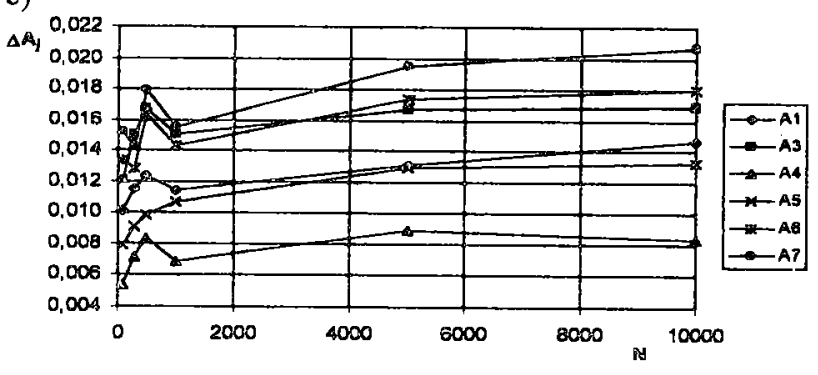

Dependence of absolute difference $\Delta A_{j}$ between batched aggregate optimal quantity maximum $A_{j \max }$ and minimum $A_{j \text { min }}$ values on the number of imitations $N$ when the ratio of $\mathrm{Mm}$ and $\mathrm{Md}$ is $\lambda: a-1 ; b-2 ; c-3$ control sieves is presented.

The area of the designed ACM grading distribution, the width of which depends on the homogeneity of used materials was obtained in the optimization model of materials quantity using statistical imitative simulation method (Monte-Carlo). This mathematical stochastic model enables to design ACM grading conforming the real conditions of its production better; therefore its accuracy is considerably higher than that of the determined models.

2. The constructed ACM designing mathematical model of aggregate optimal quantity is stable since the calculated maximum values $A_{j \max }$ of their quantity differ from minimum values $A_{j \text { min }}$ (when using average values $\sigma_{i j v i d}$ of average standard deviations of partial residues on control sieves), and their difference $\Delta A_{j}$ varies from 0,4 to $2,1 \%$ (absolute values). When values $\sigma_{i j}$ increase, values $\Delta A_{j}$ increase at the same rates.

When the number of imitations $N$ is increased from 100 to 10000 , difference $\Delta A_{j}$ increases rapidly at the beginning, but after a certain $N$, having reached the maximum values, it almost does not change. The number of imitations $N$ at which $\Delta A_{j}$ stops increasing is considered sufficient when calculating the homogeneity of the designed ACM grading.

3. Due to the obtained sufficient stability the constructed new optimization mathematical model may be used in practice using the best aggregate mass percentage used to produce ACM. The application of this model enables to calculate not only average coordinates of ACM aggregate grading curve, but also the variation interval, and from it the probability number of separate values not conforming to the requirements of norm $R 35-01$ from grading arithmetic means of aggregate estimated through the experiment and factual values (average standard deviations) of its heterogeneity.

\section{References}

I. Aivazian, S. A. Essentials of econometrics. Vol 2. Textbok (Основы эконометрики. Том 2). Moscow: Unity, 2001. 432 p. (in Russian).

2. Handy, A. Taha. Operations Research an Introduction. Upper Sadde River. New Jersy, 07458, 1997. 912 p.

3. Aksomaitis, A. Probability theory and statistics (Tikimybiu teorija ir statistika.) Kaunas: Technologija, 2000. 347 p. (in Lithuanian).

4. Zavadzkij, J. V. Task solution of motor transport by the method of imitative simulation (Репгение задач автомобильного транспорта методом имитационного моделирования). Москва: Transport, 1977. 73 с. (in Russian).

5. Cooper, K. E. Development of a practical method for the design of hot mix asphalt Transp. Res. Record 1317, Trans. Res. Board, Washington DC, 1991 p. 42-51. 
Table 5. Values of aggregate optimal quantity in the designed ACM, using average values of average standard deviation $\sigma_{i j v i d}$ of partial residues on control sieves

\begin{tabular}{|c|c|c|c|c|c|c|c|c|}
\hline \multirow[t]{2}{*}{$\lambda$} & \multirow{2}{*}{$\begin{array}{l}\text { Imitation } \\
\text { number }\end{array}$} & \multirow{2}{*}{$\begin{array}{l}\text { Maximum and } \\
\text { minimum value }\end{array}$} & \multicolumn{6}{|c|}{ Mark of aggregate $A_{j}$} \\
\hline & & & $A_{1}$ & $\mathbf{A}_{3}$ & $\mathbf{A}_{4}$ & $\mathrm{~A}_{3}$ & $A_{6}$ & $A_{7}$ \\
\hline \multirow{13}{*}{1} & \multicolumn{2}{|c|}{ Average optimal quantity } & 0,0821956 & 0,319774 & 0,164903 & 0,122901 & 0,117736 & 0,192490 \\
\hline & \multirow{2}{*}{$N=100$} & $A_{j \min }$ & 0,07656 & 0,31505 & 0,16286 & $0,1177 \overline{2}$ & 0,11131 & 0,18480 \\
\hline & & $A_{j \max }$ & 0,08693 & 0,32483 & 0,16722 & 0,12626 & 0,12391 & 0,19981 \\
\hline & \multirow{2}{*}{$N=300$} & $A_{j \min }$ & 0,07736 & 0,31376 & 0,16156 & 0,11776 & 0,11070 & 0,18585 \\
\hline & & $A_{j \max }$ & 0,08664 & 0,32476 & 0,16834 & 0,12816 & 0,12399 & 0,19984 \\
\hline & \multirow{2}{*}{$N=500$} & $A_{j \min }$ & 0,07679 & 0,31404 & 0,16136 & 0,11892 & 0,11110 & 0,18440 \\
\hline & & $A_{j \max }$ & 0,08788 & 0,32595 & 0,16874 & 0,12708 & 0,12464 & 0,19945 \\
\hline & \multirow{2}{*}{$N=1000$} & $A_{j \text { min }}$ & 0,07615 & 0,31342 & 0,16118 & 0,11792 & 0,10923 & 0,18463 \\
\hline & & $A_{\text {m max }}$ & 0,08931 & $\overline{0,32726}$ & 0,16909 & 0,12835 & 0,12558 & 0,20063 \\
\hline & \multirow{2}{*}{$N=5000$} & $A_{j \min }$ & 0,07543 & 0,31248 & 0,16060 & 0,11661 & 0,10850 & 0,18378 \\
\hline & & $A_{j \max }$ & 0,08965 & 0,32859 & 0,16844 & 0,12881 & 0,12648 & 0,20231 \\
\hline & \multirow{2}{*}{$N=10000$} & $A_{j \min }$ & 0,07528 & 0,31154 & 0,16078 & 0,11720 & 0,10862 & 0,18386 \\
\hline & & $A_{j \max }$ & 0,08903 & 0,32880 & 0,16922 & 0,12958 & 0,12690 & 0,20228 \\
\hline \multirow{13}{*}{2} & \multicolumn{2}{|c|}{ Average optimal quantity } & 0,0806701 & 0,321479 & 0,164684 & 0,122907 & 0,117754 & 0,192506 \\
\hline & \multirow{2}{*}{$N=100$} & $A_{j \min }$ & 0,07589 & 0,31599 & 0,16184 & 0,11802 & 0,11106 & 0,18655 \\
\hline & & $\overline{A_{j \max }}$ & 0,08607 & 0,32634 & 0,16726 & 0,12744 & 0,12322 & 0,19971 \\
\hline & \multirow{2}{*}{$N=300$} & $A_{j \text { min }}$ & 0,07426 & 0,31537 & 0,16093 & 0,11755 & 0,10901 & 0,18541 \\
\hline & & $A_{j \max }$ & 0,08581 & 0,32853 & 0,16893 & 0,12657 & 0,12358 & 0,20018 \\
\hline & \multirow{2}{*}{$N=500$} & $A_{j \text { min }}$ & 0,07427 & 0,31504 & 0,16124 & 0,11749 & 0,11066 & 0,18412 \\
\hline & & $A_{j \max }$ & 0,08694 & 0,32967 & 0,16705 & 0,12803 & 0,12595 & 0,20082 \\
\hline & \multirow{2}{*}{$N=1000$} & $A_{j \min }$ & 0,07415 & 0,31458 & 0,16086 & 0,11721 & 0,10825 & 0,18417 \\
\hline & & $A_{j \max }$ & 0,08785 & 0,32843 & 0,16859 & 0,12906 & 0,12426 & 0,20088 \\
\hline & \multirow{2}{*}{$N=5000$} & $A_{j \text { min }}$ & 0,07368 & 0,31409 & 0,16081 & 0,11552 & 0,10917 & 0,18270 \\
\hline & & $A_{j \max }$ & 0,08754 & 0,33004 & 0,16889 & 0,12847 & 0,12640 & 0,20191 \\
\hline & \multirow{2}{*}{$N=10000$} & $A_{j \min }$ & 0,07373 & 0,31291 & 0,16059 & 0,11612 & 0,10854 & 0,18222 \\
\hline & & $\overline{A_{j \max }}$ & 0,08836 & 0,33026 & 0,16888 & 0,12845 & 0,12757 & 0,20367 \\
\hline \multirow{13}{*}{3} & \multicolumn{2}{|c|}{ Average optimal quantity } & 0,0799283 & 0,322309 & 0,164577 & 0,122910 & 0,117762 & 0,192513 \\
\hline & \multirow{2}{*}{$N=100$} & $A_{j \min }$ & 0,07542 & 0,31557 & 0,16193 & 0,11884 & 0,11181 & 0,18636 \\
\hline & & $A_{j \max }$ & 0,08557 & 0,32768 & 0,16733 & 0,12671 & 0,12517 & 0,20159 \\
\hline & \multirow[b]{2}{*}{$N=300$} & $A_{j \text { min }}$ & 0,07497 & 0,31374 & 0,16095 & 0,11864 & 0,11158 & 0,18587 \\
\hline & & $A_{j \max }$ & 0,08648 & 0,32879 & 0,16810 & 0,12774 & 0,12442 & 0,20007 \\
\hline & $N=500$ & $A_{j \text { min }}$ & 0,07402 & 0,31381 & 0,15959 & 0,11778 & 0,11034 & 0,18246 \\
\hline & $N=500$ & $A_{j \max }$ & 0,08634 & 0,33053 & 0,16799 & 0,12764 & 0,12670 & 0,20037 \\
\hline & & $A_{j \text { min }}$ & 0,07453 & 0,31415 & 0,16134 & 0,11786 & 0,11011 & 0,18485 \\
\hline & $N=1000$ & $A_{j \max }$ & 0,08593 & 0,32919 & 0,16820 & 0,12854 & 0,12443 & 0,20040 \\
\hline & & $A_{\text {/min }}$ & 0,07335 & 0,31351 & 0,15990 & 0,11658 & 0,10974 & 0,18316 \\
\hline & $N=5000$ & $A_{j \max }$ & 0,08646 & 0,33024 & 0,16885 & 0,12950 & 0,12709 & 0,20263 \\
\hline & & $A_{j \min }$ & 0,07328 & 0,31363 & 0,16041 & 0,11597 & 0,10849 & 0,18277 \\
\hline & $N=10000$ & $A_{j \max }$ & 0,08793 & 0,33049 & 0,16877 & 0,12928 & 0,12648 & 0,20349 \\
\hline
\end{tabular}


6. Brown, S. F. Aplication of new concepts in asphalt mix design. I. Ass. Asphalt Paving Techn., 1991, 60, p. 264 286.

7. Vislavičius, K. General principles of modelling physicalmechanical properties of conglomerates. Civil Engineering (Statyba). Vol VI, No 3, Vilnius: Technika, 2000, p. 175 178 (in Lithuanian).

8. Sargious, M.; Shehata, M. Thinner pavements designed using recycled asphalt. Australian Road Research, 16(2), June 1986, p. $75-80$.

9. Sivilevičius, H.; Podviezko, V. Mathematical optimization model for filler composition in an asphalt concrete mixture. Transport Engineering (Transportas), 2001, Vol XVI, No 5, p. 167-174 (in Lithuanian).

10. Sivilevičius, H.; Podviezko, V. Conditional optimization mathematical model of the asphalt concrete mixture grading. Journal of Civil Engineering and Management, 2002, Vol VIII, No 2, p. 125-132.
11. Guideline of construction R35-10. Asphalt concrete and grit cover automobile roads (Statybos rekomendacijos R3501. Automobilių kelių asfaltbetonio ir žvyro dangos). Vilnius: LAKD. 2001. 117 p. (in Lithuanian).

12. Construction technical regulation STR 2.06.03:2001. Regulation of specifications. Roads (Statybos techninis reglamentas STR 2.06.03:2001. Techniniu reikalavimu reglamentas. Automobiliu keliai). Vilnius: LR aplinkos ministerija ir susisiekimo ministerija, 2002. 50 p. (in Lithuanian).

13. Sivilevičius, H. The quality improvement system of asphalt concrete mixture production technological process. Summary of research report presented for habilitation. Vilnius: Technika, 2003. $37 \mathrm{p}$.

14. Belevičius, R.; Kutas, R. Fortran (Fortranas). Vilnius Lietuvos mokslų akad. 1-kla, 200. 238 p. (in Lithuanian). 